\title{
ALMOST DISJOINT REFINEMENT OF FAMILIES OF SUBSETS OF $N$
}

\author{
BOHUSLAV BALCAR AND PETER VOJTÁŚ
}

\begin{abstract}
Without any set-theoretic assumptions, we prove that every uniform ultrafilter on the set $N$ of all natural numbers has a Comfort system, that is, an almost disjoint refinement. Moreover, we describe one type of ideal such that the family of all subsets of $N$ that are not contained in it has an almost disjoint refinement.
\end{abstract}

1. The problem and the theorems. For a cardinal number $\nu>1$, Hechler has generalized Pierce's notion of a $\nu$-point to a $\nu$-set of a topological space. A nonempty subset $S$ of a topological space $X$ is called a $\nu$-set if there exists a family of $\nu$ pairwise disjoint open sets, each of which contains $S$ in its closure. A point $p \in X$ is a $\nu$-point of $X$ if the singleton $\{p\}$ is a $\nu$-set. We concentrate on the space $\beta N-N=N^{*}$ of uniform ultrafilters on the set $N$ of all natural numbers.

The problem whether each point of $N^{*}$ is a $2^{\omega}$-point or, more generally, whether each nowhere dense subset of $N^{*}$ is a $2^{\omega}$-set, has a little longer history (cf. Pierce [P], Hindman [H], Comfort [CH], van Douwen [vD], Roitman [R], Kunen [K], Szymanski [Sz], Hechler [H], Frankiewicz [BF] and others). Short historical remarks can be found in $[\mathbf{C H}]$ or $[\mathbf{B F}]$.

1.1 Without any additional set-theoretic assumptions, we shall prove that every point of $N^{*}$ is a $2^{\omega}$-point. This gives an affirmative answer to a problem raised by Comfort and Hindman [CH]. We also describe a type of nowhere dense subsets of $N^{*}$ that are $2^{\omega}$-sets. The main problem of Hechler's paper [H], whether every nowhere dense subset of $N^{*}$ is a $2^{\omega}$-set, remains open.

1.2. For a set $A$ let $[A]^{\omega}$ be the set of all denumerable subsets of $A$; the notation $A \subseteq \subseteq^{*} B$ means that $A-B$ is finite. We say that a family $\left\{A_{\alpha}: \alpha<\nu\right\}$ of subsets of a set $X$ is a tower on $X$ of length $\nu$ if $A_{\alpha} \subseteq{ }^{*} A_{\beta}$ for $\alpha>\beta$. We say that a set $C$ is a selector of a family $\left\{q_{n}: n \in \omega\right\}$ if $C$ is infinite, $C \subseteq \cup\left\{q_{n}: n \in \omega\right\}$, and $\left|C \cap q_{n}\right|$ $<1$ for $n \in \omega . \mathscr{G}_{F}$ denotes the ideal of all finite subsets of $N$. In this paper all ideals are assumed to be proper and to contain $G_{F}$. Sets from $G^{+}=\mathscr{P}(N)-G$ are called large sets with respect to $G$ for an ideal $G$.

1.3. We shall deal with families $\mathbb{Q} \subseteq[N]^{\omega}$ and we look for $\mathbb{Q}$ that have an almost disjoint refinement (ADR) i.e. a family $\left\{C_{X}: X \in \mathbb{Q}\right\}$ such that

(i) $C_{X} \in[X]^{\omega}$,

(ii) for $X \neq Y$ the set $C_{X} \cap C_{Y}$ is finite.

Received by the editors February 9, 1979 and, in revised form, July 16, 1979.

AMS (MOS) subject classifications (1970). Primary 04A20; Secondary 54D35.

Key words and phrases. Ultrafilter, almost disjoint family, $2^{\omega}$-point, refinement. 
Recall that $\mathscr{P} \subseteq[N]^{\omega}$ is a MAD family on $N$ if $\mathscr{P}$ is an infinite maximal family of pairwise almost disjoint infinite subsets of $N$.

The following facts are well known.

(i) A family $\mathbb{Q} \subseteq[N]^{\omega}$ has an ADR iff there is a MADF $\mathscr{P}$ such that for every $A \in \mathbb{Q}$ we have

$$
\mid\{X \in \mathcal{P}: X \cap A \text { is infinite }\} \mid=2^{\omega} .
$$

(ii) Let $थ$ be a uniform ultrafilter on $N$. Then $\mathcal{Q}$ as a point of $N^{*}$ is a $2^{\omega}$-point of $N^{*}$ iff there is an ADR for $\mathscr{U}$.

1.4. Following Mathias $[\mathrm{M}]$ we shall say that an ideal $G$ on $N$ is tall if for all $X \in[N]^{\omega}$ there is a $Y \in[X]^{\omega}$ with $Y \in \mathscr{G}$. Let $\mathscr{P}$ be a MAD family; then $\mathscr{G}(\mathscr{P})$ denotes the ideal generated by $\mathcal{G}_{F} \cup \mathcal{P}$.

Definition. Let $Q=\left\{q_{n}: n \in \omega\right\}$ be a partition on $N$ into infinitely many (finite or infinite) pieces such that for all $k \in \omega$ there are infinitely many $q_{n}$ with at least $k$ elements. Let $\mathcal{Y}(Q)$ be the ideal generated by the union of the sets $\{X \subseteq N$ : $\left.(\exists k)(\forall n \in \omega)\left(\left|X \cap q_{n}\right|<k\right)\right\}$ and $\left\{q_{n}: n \in \Omega\right\}$. In [M] it is shown that the ideals $\mathscr{G}(\mathscr{P})$ and $\mathcal{Y}(Q)$ are both tall. It is easily seen that:

(a) If $\mathcal{Q}$ is a family with an ADR, then for every $X \in[N]^{\omega}$ there is $Y \in[X]^{\omega}$ such that $[Y]^{\omega} \cap \mathbb{Q}=\varnothing$.

(b) If $\mathbb{Q}$ is a family with an ADR then there is a MAD family $\mathscr{P}$ such that $\mathbb{Q} \subseteq \mathcal{G}^{+}(\mathscr{P})$.

(c) Tall ideals correspond to open dense subsets of $N^{*}$ that are not the whole space. If $\mathscr{G}$ is a tall ideal then $\mathcal{G}^{+}$has an ADR iff the complement of the open set corresponding to 9 is a $2^{\omega}$-set.

(d) The extremal problem whether for every MADF $\mathscr{P}$ there exists an ADR for $g^{+}(\mathscr{P})$ is equivalent to the above-mentioned problem of Hechler.

1.5. Theorem A. Let $Q$ be a partition of $N$ as in Definition 1.4. Then the family $\mathcal{Y Y}^{+}(Q)$ has an $A D R$.

As a straightforward corollary we obtain that every nonselective uniform ultrafilter on $N$ has an ADR. For ultrafilters however we shall prove a little more.

1.6. THEOREM B. Let $\mathcal{F}$ be a uniform ultrafilter on $N$ and $\mathscr{P}$ a MAD family of $N$ with $\mathscr{P} \cap \mathcal{F}=\varnothing$. Then there is an $A D R$ for $\mathscr{F}$ which consists of large sets with

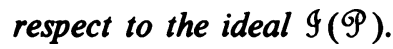

1.7. Corollary. Since the ideal $\mathscr{F}^{*}$ dual to a uniform ultrafilter $\mathscr{F}$ is tall, there is a MADF $\mathscr{P} \subseteq \mathcal{F}^{*}$. Thus every uniform ultrafilter $\mathcal{F}$ has an $A D R$.

ACKNowledgement. The authors would like to express their gratitude to $\mathbf{M}$. Dušek, whose support and understanding made this research possible, and to $P$. Simon for stimulating discussions.

2. Proofs of the theorems. We begin with some cardinal characteristics.

2.1. For functions from $N$ to $N$ consider the preordering $f<^{*} g$ iff $\{n$ : $f(n)>g(n)\}$ is finite. The least cardinal of a family of functions that is unbounded under $<^{*}$ is denoted by $\lambda$. Obviously there is a family of functions $\left\{f_{\alpha}: \alpha \in \lambda\right\}$ 
unbounded under $<^{*}$ such that $f_{\alpha}$ 's are increasing and $\alpha<\beta$ implies $f_{\alpha}<f_{\beta}$. Note that every set of functions with cardinality less than $\lambda$ has an $<*$-upper bound. Due to this fact, for every partition $\left\{X_{n}: n \in \omega\right\}$ of $N$ whose members are infinite, there is a tower $\left\{A_{\alpha}: \alpha \in \lambda\right\}$ such that

(i) $X_{n}-A_{\alpha}$ is finite for every $n, \alpha$;

(ii) for any $X \in[N]^{\omega}$, if $\left\{n:\left|X_{n} \cap X\right|=\aleph_{0}\right\}$ is infinite, then $(\exists \alpha \in \lambda)\left(X Z^{*} A_{\alpha}\right)$.

2.2. The following is defined in [BPS]. Let $\kappa$ denote the least cardinal such that the Boolean algebra $\mathscr{P}(\omega) / \mathscr{G}_{F}$ of all subsets of $\omega$ modulo finite sets is not $(\kappa, \infty)$-distributive. The "Base matrix theorem" proved in [BPS] says: There is a system $\left\{\mathscr{P}_{\alpha}: \alpha \in \kappa\right\}$ of MAD families such that for $\alpha>\beta, \mathscr{P}_{\alpha}{ }^{*}$-refines $\mathscr{P}_{\beta}$ and for every $A \in[\omega]^{\omega}$ there is a $B \in \cup\left\{\mathscr{P}_{\alpha}: \alpha \in \kappa\right\}$ such that $B \subseteq A$.

2.3. Let $d$ denote the minimal cardinal such that there is a MADF $\mathscr{P}$ on $N$ with $|\mathscr{P}|=d$.

\section{LeMMA. $\omega_{1}<\kappa<d$.}

Proof. In [BPS] the inequalities $\omega_{1}<\kappa<\lambda$ are proved. The proof is finished by adding the known inequality $\lambda<d$; see [So].

Remember that under the assumption $d=2^{\omega}$ Hechler's conjecture is known to be true [R], [H].

2.4. The following lemma plays a key role in our proofs.

Lemma. There is a family $\mathscr{B} \subseteq[\omega]^{\omega}$ such that the following conditions hold for any $u, v \in \mathscr{B}$.

(i) $u \cap v=^{*} \varnothing$ or $u \subseteq \subseteq^{*}$ or $v \subseteq^{*} u$;

(ii) $\left|\left\{w \in \mathscr{B}: u \subseteq^{*} w\right\}\right|<\kappa$;

(iii) for any $X \in[\omega]^{\omega}$ there is $a w \in B$ such that $w \subseteq X$.

Proof. Let $\left\{\mathscr{P}_{\alpha}: \alpha<\kappa\right\}$ be the base matrix mentioned in 2.2. Then $\mathscr{B}=$ $\cup\left\{\mathscr{P}_{\alpha}: \alpha<\kappa\right\}$ has the desired properties.

2.5. Lemma. Assume $\mathscr{B}^{\mathrm{B}}$ is as in Lemma $2.4, \mathscr{B}_{0} \subseteq \mathscr{B}$ such that $\left|\mathscr{B}_{0}\right|<2^{\omega}$. Then for every $C \in[\omega]^{\omega}$ there is $a u \in \mathscr{B}-\mathscr{B}_{0}$ such that $u \subseteq C$ and $\left(\forall v \in \mathscr{B}_{0}\right)(v \cap u$ $={ }^{*} \varnothing$ or $\left.u \subseteq{ }^{*} v\right)$.

Proof. As there is a MAD family on $\omega$ of cardinality $2^{\omega}$, by (iii) of Lemma 2.4 we have $\left|\left\{v \in \mathscr{B}: v \subseteq^{*} u\right\}\right|=2^{\omega}$ for every $u \in \mathscr{B}$. This fact with (i) of 2.4 finishes the proof.

2.6. Proof of Theorem A. Let $Q=\left\{q_{n}: n \in \omega\right\}$ be a partition of $N$ as in Definition 1.4. Put $\left\{A_{\alpha}: \alpha<2^{\omega}\right\}=\mathcal{Y}^{+}(Q)$. For $A_{\alpha}$ we shall now pick a set $c(\alpha)$ as follows. If $X=\left\{i \in \omega:\left|q_{i} \cap A_{\alpha}\right|=\aleph_{0}\right\}$ is infinite then we put $c(\alpha)=X$. Otherwise we pick $c(\alpha) \in[\omega-X]^{\omega}$ such that $i<j$ implies $\left|A_{\alpha} \cap q_{i}\right|<\left|A_{\alpha} \cap q_{j}\right|$ for $i, j \in c(\alpha)$. Let $\mathscr{B}$ be the Base family from Lemma 2.4. In the sequel $\mathscr{B}$ is used on $\omega$ as indexes of $\left\{q_{i}: i \in \omega\right\}$. By transfinite recursion through $\alpha<2^{\omega}$ we shall define $F(\alpha) \in\left[A_{\alpha}\right]^{\omega}$ and $I(\alpha) \subseteq c(\alpha)$ such that

(i) $I(\alpha) \in \mathscr{B}$;

(ii) $F(\alpha)$ is a selector for $R_{\alpha}=\left\{q_{i} \cap A_{\alpha}: i \in I(\alpha)\right\}$ i.e. $F(\alpha) \subseteq \cup R_{\alpha}$ and $\left|F(\alpha) \cap q_{i} \cap A_{\alpha}\right|<1$ for any $i \in I(\alpha)$; 
(iii) $F(\alpha)$ is almost disjoint with all $F(\beta)$, and $I(\alpha) \neq^{*} I(\beta)$ for $\beta<\alpha$.

For $\alpha<2^{\omega}$ we put $D_{\alpha}=\cup R_{\alpha}$.

Step 0 . There is $I(0) \in \mathscr{B}$ such that $I(0) \subseteq c(0)$. As $F(0)$ take an (infinite) selector of $R_{0}$.

Step $\alpha<2^{\omega}$. For $\beta, \gamma<\alpha$ we have $F(\beta), I(\beta)$ such that $I(\beta) \neq \neq^{*} I(\gamma)$ and $F(\beta) \cap F(\gamma)$ is finite for $\beta \neq \gamma$. Choose $I(\alpha)$ using 2.5 with respect to $\mathscr{B}_{0}=\{I(\beta)$ : $\beta<\alpha\}$ and *-different from all $I(\beta)$. Put $\mathcal{V}=\left\{F(\beta) \cap D_{\alpha}: \beta<\alpha\right.$ and $I(\alpha)$ $\subseteq^{*} I(\beta)$ and $\left.\left|F(\beta) \cap D_{\alpha}\right|=\kappa_{0}\right\} \cup\left(R_{\alpha} \cap[N]^{\omega}\right)$.

Members of $\mathcal{V}$ are pairwise almost disjoint. According to the choice of $c(\alpha)$ and since $F(\beta)$ 's are selectors, $D_{\alpha}$ cannot be $={ }^{*}$ to the union of any finite part of $\mathcal{V}$. By (ii) of Lemma 2.4 we have $|\mathscr{V}|<\kappa \leqslant d$. Hence there is $F(\alpha) \subseteq D_{\alpha}$, an infinite selector of $R_{\alpha}$ that is almost disjoint with all members of $\mathfrak{T}$. We note that $I(\alpha) \cap I(\beta)={ }^{*} \varnothing$ implies $F(\alpha) \cap F(\beta)={ }^{*} \varnothing$. Hence $\left\{F(\alpha): \alpha<2^{\omega}\right\}$ is an ADR for $\mathscr{Y}^{+}(Q)$. The proof of Theorem $A$ is complete.

2.7. Our starting point for the proof of Theorem B is the notion of an ultrafilter's tower.

Definition. A tower $\mathfrak{A}=\{A(\alpha): \alpha \in \nu\}$ is a tower of a uniform ultrafilter $\mathscr{F}$ if

(i) $\nu$ is uncountable and regular;

(ii) $\mathfrak{A} \subseteq \mathscr{F}$;

(iii) for any $X \in \mathscr{F}$ there is $\alpha \in \nu$ such that $X \mathbb{Z}^{*} A(\alpha)$.

2.8. Lemma. Assume $\mathcal{F}$ is a uniform ultrafilter on $N$. Then there is a tower of $\mathcal{F}$ (of uncountable length).

Proof. It is clear that such a tower exists for $P$-ultrafilters. In the case of non- $P$-ultrafilters we can take a tower of the length $\lambda$ from 2.1, where the partition is the one exemplifying the non-P-property.

2.9. LemMA. Assume $\{A(\alpha): \alpha \in \nu\}$ is a tower of $\mathscr{F}$ and $\mathscr{P}$ is a MAD family such that $\mathscr{F} \cap \mathcal{P}=\varnothing$. Then

(iv) $(\forall \alpha \in \nu)(\exists \beta>\alpha)\left(A(\alpha)-A(\beta) \in \mathscr{G}^{+}(\mathcal{P})\right)$.

Proof. By induction we can choose an increasing sequence $\left\{\alpha_{n}: n \in \omega\right\}$ and a family $\left\{u_{n}: n \in \omega\right\}$ of different elements of $\mathscr{P}$ such that $\left(A\left(\alpha_{i}\right)-A\left(\alpha_{i+1}\right)\right) \cap u_{i}$ is infinite. For $n \in \omega$ we have $A\left(\alpha_{n}\right)-\cup\left\{u_{i}: 0<i<n-1\right\} \in \mathscr{F}$. Hence by (iii) of Definition 2.7 there are $\alpha_{n+1}>\alpha_{n}$ and $u_{n} \in \mathcal{P}-\left\{u_{0}, \ldots, u_{n-1}\right\}$ such that $\left(A\left(\alpha_{n}\right)\right.$ $\left.-A\left(\alpha_{n+1}\right)\right) \cap u_{n}$ is infinite. Put $\beta=\sup \left\{\alpha_{n}: n \in \omega\right\}$.

2.10. Proof OF TheORem B. Let $\mathscr{F}$ be a uniform ultrafilter and $\mathscr{P}$ a MADF with $\mathscr{P} \cap \mathscr{F}=\varnothing$. Assume $\{A(\alpha): \alpha<\nu\}$ is a tower of $\mathscr{F}$ with $A(\alpha)-A(\beta) \in \mathscr{F}^{+}(\mathscr{P})$ for $\alpha<\beta$. We put $\nu(\omega)=\{\alpha \in \nu$ : $\operatorname{cf}(\alpha)=\omega\}$. For every $\alpha \in \nu(\omega)$ we fix an increasing sequence $\left\{\alpha_{n}: n \in \omega\right\}$ such that $\alpha=\sup \left\{\alpha_{n}: n \in \omega\right\}$. We define $q(\alpha, n)$ $=\cap\left\{A\left(\alpha_{i}\right): 0<i<n\right\}-\left(A\left(\alpha_{n+1}\right) \cup A(\alpha)\right)$. Note that $q(\alpha, n) \in \mathscr{I}^{+}(\mathcal{P})$ and $q(\alpha, n) \cap q(\alpha, m)=\varnothing$ for all $\alpha, n \neq m$. It is easy to see by Lemma 2.9 that for every $X \in \mathscr{F}$ there is $\alpha \in \nu(\omega)$ such that the set $\left\{n: X \cap q(\alpha, n) \in \mathscr{I}^{+}(\mathscr{P})\right\}$ is infinite. 
We define $Q_{\alpha}=\{q(\alpha, n): n \in \omega\}$ for $\alpha \in \nu(\omega)$. If $K_{\alpha}, K_{\beta}$ are selectors of $Q_{\alpha}, Q_{\beta}$ respectively and $\alpha \neq \beta$ then $K_{\alpha} \cap K_{\beta}$ is finite. Hence for the proof of Theorem B it suffices to show that the family $\delta\left(Q_{\alpha}\right)=\left\{X \subseteq N:\left|\left\{n: q(\alpha, n) \cap X \in \mathcal{G}^{+}(\mathcal{P})\right\}\right|=\right.$ $\left.\aleph_{0}\right\}$ has an ADR consisting of selectors of $Q_{\alpha}$ which are large sets. The argument is now similar to the one used in 2.6. Let $Q=\left\{q_{n}: n \in \omega\right\}=Q_{\alpha}$ for $\alpha \in \nu(\omega)$ and let $\left\{D(\alpha): \alpha<2^{\omega}\right\}$ be a numbering of $\delta(Q)$. We put $c(\alpha)=\left\{i: D(\alpha) \cap q_{i} \in\right.$ $\left.g^{+}(\mathscr{P})\right\}$. By transfinite recursion we define sets $I(\alpha), F(\alpha)$ such that

(i) $I(\alpha) \in \mathscr{B}$, where $\mathscr{B}$ is the Base family from Lemma 2.4 and $I(\alpha) \subseteq c(\alpha)$;

(ii) $F(\alpha)$ is a selector for $\left\{q_{i} \cap D_{\alpha}: i \in I(\alpha)\right\}$ and $F(\alpha) \in \mathscr{I}^{+}(\mathcal{P})$;

(iii) for $\beta<\alpha, F(\alpha) \cap F(\beta)$ is finite and $I(\beta) \cap I(\alpha)={ }^{*} \varnothing$ or $\left(I(\alpha) \subseteq \subseteq^{*} I(\beta)\right.$ and $\left.I(\alpha) \neq^{*} I(\beta)\right)$.

In the step $\alpha<2^{\omega}$ we choose $I(\alpha) \in \mathscr{B}$ using Lemma 2.5. The $F(\beta)$ 's are selectors and hence they determine partial functions $f_{\beta}$ on $\omega$. We set $\mathcal{V}=\left\{f_{\beta} \cap\right.$ $(I(\alpha) \times D(\alpha)): \beta<\alpha$, and $\left.I(\alpha) \subseteq^{*} I(\beta)\right\}$. As $|\mathfrak{V}|<\kappa<\lambda$ there is a function $f$ : $I(\alpha) \rightarrow N$ that is an $<*$-upper bound for $\mathscr{V}$. We note that for any infinite family of pairwise disjoint large sets there is a large selector. Hence we may take $F(\alpha)$ as a large selector of the family $\left\{D_{\alpha} \cap q_{i}-\{n: n<f(i)\}: i \in I(\alpha)\right\}$. It is obvious that $F(\alpha) \cap F(\beta)$ is finite for $\beta<\alpha$.

This completes the proof.

\section{Remarks and problems.}

3.1. We do not know if the following observation is known. Let us consider a MAD family on the set $Q$ of all rational numbers in the unit interval $[0,1]$ of the real line. Then there is a MAD family $\mathscr{P}$ on $Q$ such that for any set $A \subseteq Q$ that has infinitely many accumulation points in the space $[0,1]$ there exists $B \in \mathcal{P}$ with $B \subseteq A$. This fact follows from Theorem $\mathrm{A}$.

3.2. Let $s=\left\{a_{n}: n \in N\right\}$ be a sequence of positive reals with $\lim a_{n}=0$ and $\Sigma a_{n}=\infty$. Let us consider the ideal $\mathcal{Y}(s)=\left\{X \subseteq N: \Sigma\left\{a_{n}: n \in X\right\}<\infty\right\}$. This type of ideal seems to be similar to the ideal of type $\mathscr{Y}(Q)$ from Definition 1.4, where $Q$ is partition of $N$ consisting of finite sets. But we do not know whether $\mathrm{Y}^{+}(s)$ has an ADR.

3.3. Does the assumption "every $<{ }^{*}$-cofinal subset of functions from $N$ to $N$ has cardinality $2^{\omega}$ " imply Hechler's conjecture?

3.4. Consider the Boolean algebra $B=\mathcal{P}(N) / \mathscr{G}_{F}$. Corollary 1.7 is equivalent to the statement "every filter base on $B$ of cardinality at most $2^{\omega}$ has a disjoint refinement". This statement cannot be strenghtened to the completion $\bar{B}$ of the algebra $B$. Using a result of Kunen, van Mill and Mills [KvMM] in [BSV] have proved the following.

If $2^{\tau}<2^{\omega}$ for all $\tau<2^{\omega}$ then there is an ultrafilter on $\bar{B}$ with a base of cardinality $2^{\omega}$. Then there is no disjoint refinement on $\bar{B}$ for any base of this ultrafilter.

3.5. K. Kunen, using an observation from [BF], proved the following generalization of a result in [BF]. If $X$ is any compact space in which nonempty $G_{\delta}$ sets have nonempty interior, then very nonisolated point in $X$ is an $\omega_{1}$-point. He also has remarked that for the above class of spaces we cannot replace $\omega_{1}$ by $2^{\omega}$. 
Let us consider only spaces that moreover have no isolated point. Are there any simple conditions on such spaces that imply "every point is a $2^{\omega}$-point"? We note that for arbitrary $\tau>\omega$, every point of the space $\beta(\tau)-\tau$ is a $2^{\omega}$-point.

\section{REFERENCES}

[BF] B. Balcar and R. Frankiewicz, Ultrafilters and $\omega_{1}$-points in $\beta N-N$ (to appear).

[BSV] B. Balcar, P. Simon and P. Vojtás, Refinement properties and extending of filters in Boolean algebras (to appear).

[BPS] B. Balcar, J. Pelant and P. Simon, The space of ultrafilters on $N$ covered by nowhere dense sets, Fund. Math. (to appear).

[CH] W. W. Comfort and N. B. Hindman, Refining families for ultrafilters, Math. Z. 149 (1976), 189-199.

[vD] E. K. van Douwen, Martin's axiom and pathological points in $\beta X-X$ (manuscript).

[H] S. H. Hechler, Generalization of almost disjointness, c-sets, and the Baire number of $\beta N-N$, General Topology and Appl. 8 (1978), 93-110.

[Hd] N. B. Hindman, On the existence of c-point in $\beta N-N$, Proc. Amer. Math. Soc. 21 (1969), 277-280.

[J] T. J. Jech, Set theory, Academic Press, New York, 1978.

[K] K. Kunen, Letter to the author, Sept. 14, 1978.

[KvMM] K. Kunen, J. van Mill and Ch. F. Mills, On nowhere dense closed P-sets (to appear).

[M] A. R. D. Mathias, Happy families, Ann. Math. Logic 12 (1977), 59-111.

[P] R. C. Pierce, Modules over commutative regular rings, Mem. Amer. Math. Soc. no. 70, 1967.

[R] J. Roitman, Almost disjoint strong refinements, Notices Amer. Math. Soc. 22 (1975), A 328.

[So] R. C. Solomon, Families of sets and functions, Czechoslovak Math. J. 27 (102) (1977), 556-559.

[Sz] A. Szymanski, On the existence of $K_{0}$-points, Proc. Amer. Math. Soc. 66 (1977), 128-130.

[T] A. D. Taylor, Regularity properties of ideals and ultrafilters, Ann. Math. Logic 16 (1979), 33-55.

ČKD-Polovodiče, 14003 Prague, Czechoslovakia

Mathematical Institute of Slovac Academy of Sciences, Komenskźho 14, 04154 Košice, Czechoslovakia 